\title{
Ein spezifischer Marker für PML
}

Fragestellung: Ziel der Studie war es, den komplementären Nutzen des spezifischen JCV-Antikörperindex $\left(\mathrm{AI}_{\mathrm{JCV}}\right)$ bei der Diagnose von Natalizumab-assoziierter PML zu bewerten.

Hintergrund: Das JC-Virus (JCV) ist Ursache einer progressiven multifokalen Leukenzephalopathie (PML). Patienten mit Multipler Sklerose (MS), die mit dem monoklonalen Antikörper Natalizumab (NAT) behandelt werden, können an PML erkranken. Die PML-Diagnose erfordert den JCV-Nachweis mittels quantitativer Polymerasekettenreaktion (qPCR) im Liquor (CSF) oder eine Hirnbiopsie. Bei zu geringer JCV-Viruslast $<100$ Kopien JCV-DNA/ml CSF) kann die Diagnosestel-

Warnke C, von Geldern G, Markwerth P et al. Cerebrospinal fluid JC virus antibody index for diagnosis of natalizumabassociated progressive multifocal leukoencephalopathy. Ann Neurol 2014; 76: 792 -801 bestimmt. Seren und CSF wurden paarweise im validierten ELISA-Assay getestet [1]. Albuminspiegel und IgG-Konzentrationen wurden immunnephelometrisch bestimmt und der $\mathrm{AI}_{\mathrm{JCV}}$ standardmäßig berechnet. Ein $\mathrm{AI}_{\mathrm{JCV}}>1,5$ galt als pathologisch und sprach für eine intrathekale Anti-JCV-IgG-Produktion.

Ergebnisse: Insgesamt 70\% (26/37) der Patienten mit NATassoziierter PML hatten einen pathologischen $\mathrm{AI}_{\mathrm{ICV}}$, während dies in keiner der Kontrollen gesehen wurde ( $<<0,0001)$. Zum Zeitpunkt des ersten positiven qPCR für JCV-DNA hatten elf von 20 Patienten (55\%) mit NAT-assoziierter PML einen pathologischen $\mathrm{AI}_{\mathrm{JCV}}$. Niedrige Liquorviruslasten wurden in 14 dieser 20 Patienten (70\%) beobachtet, davon acht (57\%) mit pathologischem $\mathrm{AI}_{\mathrm{JCV}}$.

Schlussfolgerungen: Die Bestimmung des $\mathrm{AI}_{\mathrm{JCV}}$ ist spezifisch und ermöglicht unter Umständen eine frühere PML-Diagnose. Es könnte somit ein zusätzlicher Marker für die PML-Diagnostik sein und sollte in den Diagnosekriterien von NAT-assoziierter PML enthalten sein.

\section{- Kommentar von Til Menge, Düsseldorf}

\section{Mehr diagnostische Sicherheit durch $\mathrm{Al}_{\mathrm{JCV}}$-Bestimmung}

Diese Daten sind schon längst überfällig, und man muss sich fragen, warum sie jetzt erst systematisch aufgearbeitet werden konnten; schließlich wurden nur Materialien (Liquor und Serum) benötigt, die für die JCV-PCR ohnehin abgenommen wurden. Die Studie zeigt eindrücklich, dass die $\mathrm{Al}_{\mathrm{JCV}}$-Bestimmung für eine humorale Antikörperantwort gegen das JCVirus nicht nur die diagnostische Sicherheit einer PML erhöht, sondern auch zu einer rascheren Diagnosestellung bei Patienten führt, deren Viruslast im Liquor (noch) zu niedrig ist und im diagnostischen Graubereich liegt. In dieser Studie hatten $70 \%(14 / 20)$ der Patienten im ersten Liquor < 100 Viruskopien/ $\mathrm{ml}$, was in den meisten Labors unterhalb der messtechnischen Nachweisgrenze liegt. Bei vielen dieser Patienten konnte die Diagnose PML erst durch weitere Liquorpunktionen und Viruslastbestimmungen im Verlauf, teilweise erst vier Monate später, gestellt werden - wertvolle Zeit für die rasche Elimination von NAT aus dem Körper. Durch Nachweis eines pathologisch erhöhten $\mathrm{Al}_{\mathrm{Jcv}}$ wäre über die Hälfte dieser Patienten (8/14) zum Zeitpunkt der ersten Liquorpunktion korrekt als PML-Fall erkannt worden. Wohlgemerkt, auch bei nicht an PML erkrankten, mit NAT behandelten Patienten ließen sich Antikörper gegen JCV im Liquor nachweisen. Dies sind Serumantikörper, die unspezifisch in den Liquor gelangen. Denn bei keinem dieser 89 Kontrollen war der AIJCV pathologisch erhöht, sodass es sich um einen sehr spezifischen Marker handelt. Erst dieser Marker macht aus einem positiven JCV-Serostatus eine PML, quasi in Analogie zu Borreliose oder Lues, wo auch erst durch pathologische entsprechende Antikörper- indices aus systemischen Infektionserkrankungen die Diagnosen Neurolues oder Neuroborreliose werden.

Ab jetzt sollte bei allen Patienten, die aus diagnostischen Gründen unter PML-Verdacht liquorpunktiert werden, auch der $\mathrm{Al}_{\jmath \mathrm{cv}}$ bestimmt werden. Dieser Marker ist zwar nicht validiert, erhöht aber die diagnostische Sicherheit. Jedoch schließt ein negativer $\mathrm{Al}_{\mathrm{JcV}}(<1,5)$ eine $\mathrm{PML}$ nicht aus. Anzumerken ist, dass der $\mathrm{Al}_{\mathrm{JCV}}$ als ein Marker der spezifischen Antikörpersynthese gegen JCV im Liquor nicht mit den „index values“ verwechselt werden darf, die mehr oder minder einen Titer der Serumantikörper wiedergeben: je höher der "index value“, desto stärker die Antikörperreaktivität. Höhere „index values“ gehen unter NAT-Therapie möglicherweise mit einem erhöhten PML-Risiko einher [2].
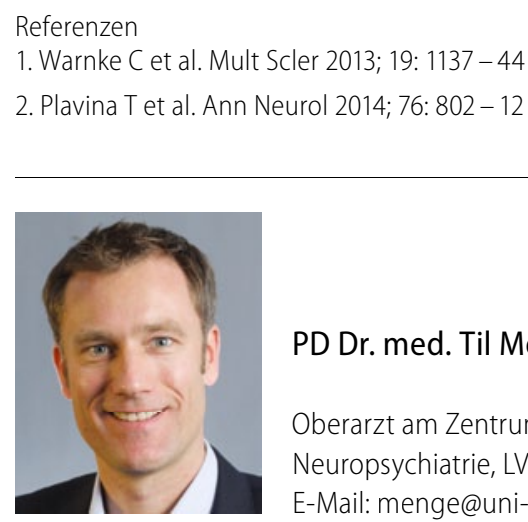

PD Dr. med. Til Menge, Düsseldorf

Oberarzt am Zentrum für Neurologie und Neuropsychiatrie, LVR-Klinikum Düsseldorf E-Mail:menge@uni-duesseldorf.de 